\title{
Features versus spatial phase in a tachistoscopic laterality experiment
}

\author{
INGO RENTSCHLER and LISANNE CHRISTEN \\ Institute of Medical Psychology, University of Munich, Munich, West Germany \\ STEPHAN CHRISTEN \\ Social Psychiatric Service, Psychiatric University Hospital, Zurich, Switzerland \\ and \\ THEODOR LANDIS \\ Department of Neurology, University Hospital, Zurich, Switzerland
}

\begin{abstract}
The abilities of the two hemispheres to instantly discriminate compound grating pairs with identical power spectra and the same separation in phase $\left(180^{\circ}\right)$ were compared in a tachistoscopic laterality experiment. Consistent with earlier observations by Fiorentini and Berardi (1984), a left-visual-field advantage was found for a stimulus pair differing in local contrast magnitude, whereas discrimination was not possible at all for a mirror-symmetric grating pair. This result contradicts the notion of a right-hemisphere advantage in processing spatial phase. Moreover, it is incompatible with the assumption that compound gratings are discriminated with respect to their Fourier components. This implies that discrimination must depend on the processing of local image components or features. Although the right-hemisphere advantage found is related to the analysis of local contrast variability within an image, the relative position of local image components is generally lost in extrafoveal vision.
\end{abstract}

Since discrimination depends greatly on the relative energies of target signals, the study of visual pattern discrimination under constant-energy conditions is of interest. Techniques for producing iso-energy patterns by varying luminance profiles are few in number and restricted in form (Caelli, Julesz, \& Gilbert, 1978). The modulation of phase (i.e., relative position) values of the spatial frequency components of stimulus patterns is less difficult (e.g., for phase quantization, see Goodman \& Silvestri, 1970, and Caelli \& Bevan, 1982; for phase shift, see Caelli, Hübner, \& Rentschler, 1985). Provided the respective amplitude values (or contrasts of the spatial frequency components) are left unaltered, an original pattern and its phase-modulated versions have identical power spectra. Thus, it would seem that the analysis of sensitivities to spatial phase modulation would allow for conclusions to be reached as to the visual processing of stimulus structure or perceived form.

This has motivated a number of studies on the discrimination of compound gratings that consist of a fundamental sinusoidal waveform and its third harmonic with variable spatial phase relationships (Atkinson \& Campbell, 1974; Burr, 1980; Gayl, Strasburger, Rentschler, \& Hilz, 1982; Lawden, 1983; Nachmias \& Weber, 1975). The

This study was supported by Grant RE 337/3-2 of Deutsche Forschungsgemeinschaft to I.R. and T.L. I. Rentschler's mailing address is: Institute of Medical Psychology, University of Munich, Goethestr. 31, D 8000 Munich 2, West Germany. same paradigm has been applied by Lawden (1982), Lawden, Hess, \& Campbell (1982), and Weiss, Rentschler, \& Caelli (1985) for analyzing the deterioration of spatial vision in amblyopia. What the results of these studies have in common is that they cannot be explained simply in terms of contrast sensitivities to isolated grating components.

The hypothesis that compound grating discrimination is not subserved by the same mechanisms that underlie the processing of simple sinusoidal gratings received support from the findings of Fiorentini and Berardi (1980, 1981) concerning perceptual learning in grating discrimination. According to those findings, the ability to discriminate compound gratings improved with training and learning was found to be selective for orientation and spatial frequency. Spatial frequency discrimination of sinusoidal gratings, however, did not improve with practice.

In a subsequent study, Fiorentini and Berardi (1984) investigated possible visual-field differences in various detection and discrimination tasks for sinusoidal gratings or compound gratings. Their results indicated an absence of hemispheric specialization for the detection and spatial frequency discrimination of sinusoidal gratings, but a leftvisual-field advantage for the discrimination of compound gratings. To Fiorentini and Berardi, this suggested a righthemisphere superiority of spatial phase processing.

Such a lateralization of spatial phase processing would be of great interest to neuropsychological research. Phase coding, by definition, determines the relative position of 
spatial frequency components and, therefore, the structure of a stimulus pattern (see Brettel, Caelli, Hilz, \& Rentschler, 1982). This is obvious from the intelligibility of phase-only reconstructions for images, speech, and crystallographic structures (Oppenheim \& Lim, 1981). Thus, evidence for a lateralization of spatial phase processing might be essential for understanding why, in tachistoscopic experiments, the right hemisphere has been found to be more competent in analyzing the spatial structure of stimulus patterns (for a review, see Beaumont, 1982).

The problem with Fiorentini and Berardi's conclusion is that they restricted their experiments to grating stimuli that differed both in the phase relationships of their harmonic components and in the magnitude of local contrasts. Thus, it is conceivable that their subjects used information about the luminance profiles of the compound gratings rather than encoding phase information (Badcock, 1984).

To directly test the effect of spatial pattern structure on the discrimination abilities of the left and right hemispheres, we conducted a tachistoscopic discrimination experiment with two pairs of stimuli which had identical power spectra and the same separation $\left(180^{\circ}\right)$ in spatial phase. The difference between the two pairs was that one pair, as in Fiorentini and Berardi's experiments, consisted of compound gratings with clearly different local features. For this pair, the appearance of the stripes differed in the contrast of a narrow dark band on a lighter gray stripe (Figure $1,0^{\circ}$ and $180^{\circ}$ ). No such difference in contrast magnitude existed for the other grating pair, in which the darker stripes were identical in quality and differed only in that their positions were mirror-image reversed in the two patterns (Figure $1,90^{\circ}$ and $270^{\circ}$ ).

Such compound grating pairs with identical power spectra have been used recently by Rentschler and Treutwein (1985) in a study that compared foveal and extrafoveal mechanisms of rapid pattern discrimination. Although each pair was discriminated equally well when seen in foveal vision, non-mirror-image pairs were discriminated much more easily than mirror-symmetrical pairs in extrafoveal vision. These results, however, were obtained by presenting the extrafoveal stimuli exclusively in the right visual field (RVF, left hemisphere). Thus, it is an open question as to whether this is a phenomenon of extrafoveal stimulus presentation as such or whether it depends on the particular hemisphere being stimulated.

\section{METHOD}

\section{Subjects}

Twenty-four right-handed male students from the University of Zürich, with normal or corrected-to-normal vision and aged 20 to 29 years, participated in the experiment.

\section{Stimuli}

The stimuli were vertical gratings generated by means of a LSI 11/23 laboratory computer on an oscilloscope screen (HP 1304, P4 phosphor). Space-average luminance was kept constant at $10 \mathrm{~cd} \mathrm{~m}^{-2}$. For presentation in a two-channel tachistoscope, the grat-

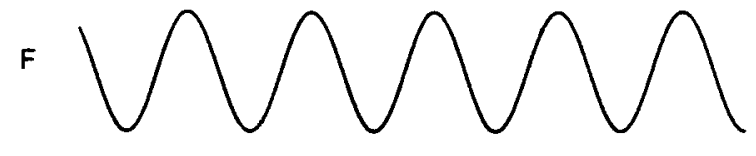

"MFMMMMMM
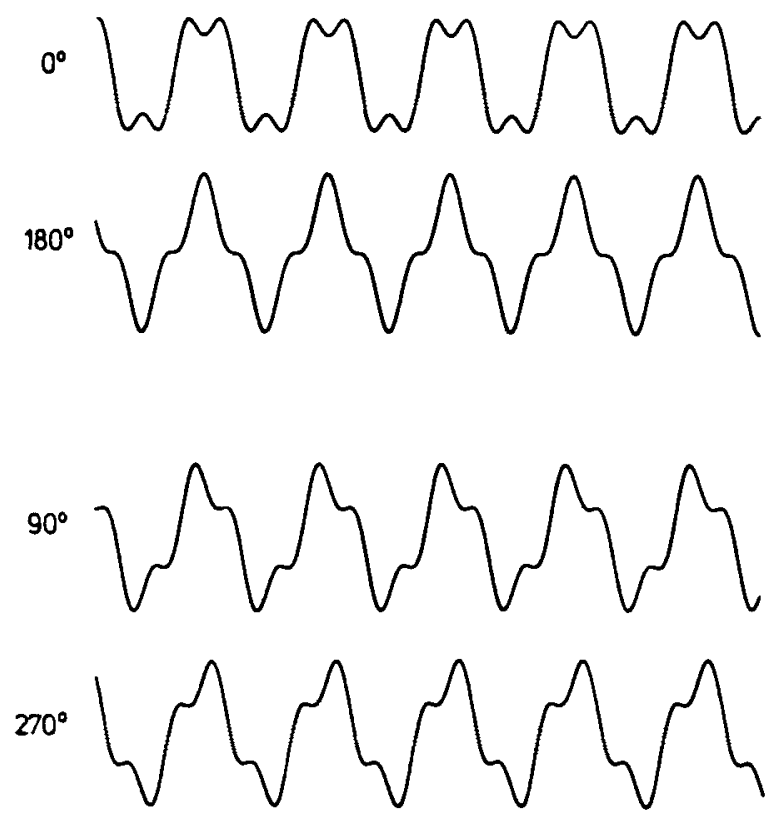

Figure 1. Luminance profiles of a fundamental (F) sinusoidal grating and its third harmonic (3F). The corresponding amplitude, that is, contrast ratio is 3:1. At the center is a pair of resulting compound grating waveforms with $0^{\circ}$ and $180^{\circ}$ phase relationships. These gratings differ in spatial contrast densities. At the bottom is a pair of gratings with $90^{\circ}$ and $270^{\circ}$ phase relationships. The latter gratings have identical spatial contrast densities, but differ in the relative positions of image components. Note that both grating pairs have identical power spectra and the same phase separation $\left(180^{\circ}\right)$ between their fundamental and third harmonic spatial frequency components.

ings were photographed using a Rollei SL 36 camera and Polaroidtype 667 film. Four compound gratings of different waveforms were used. They consisted of the sums of two sinusoidal components of spatial frequencies $F$ and $3 F$ with relative contrasts of 1 and $1 / 3$. The values of the phase relationships between these components were $0^{\circ}$ ("peaks subtract"), $90^{\circ}, 180^{\circ}$ ("peaks add"), and $270^{\circ}$ (Figure 1).

The photographic process is a nonlinear one. Thus, the relationship between CRT modulation voltage and resulting photographic density was determined by using a Spectra Pritchard Photometer (Model $1980 \mathrm{~A}$ ) and a calibration gray scale of 11 steps. This resulted in the following peak-to-trough contrast values (differences of local luminance values divided by their sum) for the four types of patterns used: $0^{\circ}, 52 \% ; 180^{\circ}, 79 \% ; 90^{\circ}, 72 \% ; 270^{\circ}, 72 \%$.

Each stimulus card for tachistoscopic presentation contained four vertical grating parterns which were all the same (nontarget cards) or of which one was mistmatched (target cards). Each of the grat- 
ing patterns subtended $4.5^{\circ}$ horizontally and $2^{\circ}$ vertically. Two of them were positioned to the left and two were positioned to the right of the fixation point. The inner edge of each grating was $2^{\circ}$ away from the vertical meridian. Within each visual half-field, the gratings were positioned symmetrically around the horizontal meridian with a $0.5^{\circ}$ vertical separation between them. The fundamental spatial frequency of the gratings was $1.2 \mathrm{cpd}$ at a viewing distance of $63 \mathrm{~cm}$, so that each pattern showed at least 3 full cycles.

In Experiment 1, we studied the discrimination of $0^{\circ}$ gratings versus $180^{\circ}$ gratings; Experiment 2 was devoted to the discrimination of $90^{\circ}$ gratings versus $270^{\circ}$ gratings. Accordingly, 48 stimulus cards were prepared for each experiment. Sixteen nontarget cards contained in all four positions either $180^{\circ}$ patterns (Experiment 1) or $270^{\circ}$ patterns (Experiment 2). Another 32 target cards had one of these patterns replaced by either a $0^{\circ}$ target (Experiment 1 ) or a $90^{\circ}$ target (Experiment 2). The target patterns occurred with equal frequencies in one of the four quadrants of the visual field.

Four additional stimulus cards were used in an auxiliary experiment. Two of these cards contained four blank fields that matched the space average luminance of the compound gratings. Another two cards had one blank field replaced by a photograph that showed the third harmonic (3F) of the compound gratings in isolation. The spatial arrangement of these cards was the same as in the main experiments.

\section{Procedure}

The subjects sat in front of a two-channel tachistoscope. They were instructed to fixate a center point as soon as they heard the warning tone that preceded stimulus exposure.

In the auxiliary experiment, it was established that the observers were able to detect the third harmonic of the compound gratings when it was presented in isolation. That is, the blank cards and those with the third harmonic were presented in random order and the subjects were instructed to report the occurrence of a mismatched pattern, which was the sinusoidal grating. This procedure was repeated at 20-, 50-, and $150-\mathrm{msec}$ exposure durations.

After successfully detecting the isolated third harmonic, the subjects were shown one nontarget card and one target card in free vision. They were required to point to the mismatched grating. The subjects were then told that they were now to see, in the tachistoscope, cards containing either four identical patterns or only three identical ones plus one mismatched pattern. They were asked to press response buttons with either hand as soon as they detected a mismatched grating, irrespective of its position.

There were three runs, one each at the 20-, 50-, and 150-msec exposure durations in both experiments. The subjects were assigned randomly to six orders of runs. Half of subjects were assigned to start with Experiment 1, and half were assigned to start with Experiment 2 .

\section{Analysis of Results}

Two-way repeated measures analyses of variance (ANOVAs) were computed on totals of correct responses with respect to the visual fields (VF) and exposure duration (E): a (VF $\times \mathrm{E} \times$ Subjects) design. Single comparisons were made by $t$ tests.

\section{RESULTS}

\section{Experiment 1: Peaks-Subtract Versus Peaks-Add Gratings}

In this experiment, the nontarget stimuli consisted of compound gratings with a peaks-add $\left(180^{\circ}\right)$ phase relationship, while the targets had a peaks-subtract $\left(0^{\circ}\right)$ phase angle. The means and standard deviations for the total numbers of correct identifications of targets for each visual field and the exposure durations are given in Table 1 . That is, for each visual half-field (VHF), the data were col-
Table 1

Discrimination of Compound Gratings with the $0^{\circ}$ and $180^{\circ}$ Phase Relationship (Experiment 1)

\begin{tabular}{cccc}
\hline & \multicolumn{3}{c}{ Exposure Durations } \\
\cline { 2 - 4 } & $20 \mathrm{msec}$ & $50 \mathrm{msec}$ & $150 \mathrm{msec}$ \\
\hline \multicolumn{4}{c}{ Correct Identifications } \\
LVF & $7.58-3.43$ & $10.50-2.78$ & $14.00-2.45$ \\
RVF & $7.96-3.59$ & $8.96-3.13$ & $12.88-3.19$ \\
& \multicolumn{3}{c}{ Response Latencies } \\
LVF & $768-280$ & $738-194$ & $665-137$ \\
RVF & $762-272$ & $720-160$ & $686-123$ \\
\hline
\end{tabular}

Note-Means and standard deviations $(\mathrm{N}=24)$ of the total number of correct identifications (top) and of response latencies (in milliseconds, bottom). For each visual half-field, data were collapsed over the upper and lower quadrants, resulting in 16 target exposures per condition and observer.

lapsed over the upper and lower quadrant, resulting in $2 \times 8=16$ replications per condition and observer. The ANOVA revealed a significant effect of VHFs $[F(1,23)$ $=4.73, p<.04]$ in favor of the left-visual field (LVF) and a significant effect of exposure duration $[F(2,46)=$ $32.11, p<.001]$. The difference was significant between 20 and $50 \mathrm{msec}[t(23)=-2.74, p<.01]$, between 20 and $150 \mathrm{msec}[t(23)=-6.36, p<.001]$, and between 50 and $150 \mathrm{msec}[t(23)=-7.50, p<.001]$. Performance improved with longer exposure durations. The interaction of VHF $\times$ exposure duration was also significant $[F(2,46)=3.32, p<.05]$, indicating an increase of the LVF advantage with longer exposure duration. Single comparisons showed a significant LVF advantage only at the 150 -msec exposure duration $[t(23)=2.39, p<.03]$. The analysis of latencies of correct identifications revealed no significant main effects (VHF, $p=1.0$; exposure durations: $p=.07)$ or interaction $(p=.4)$. Responses were fastest at the 150 -msec conditions.

In summary, the discrimination of sinusoidal gratings with the $0^{\circ}$ and $180^{\circ}$ phase angles between their first and third harmonic was significantly more accurate in the LVF (right hemisphere). This effect was most pronounced at the 150-msec exposure duration. An increase in performance with increasing exposure durations was obtained in both VHFs. Response latencies did not differ significantly.

\section{Experiment 2: Discrimination of Mirror-Symmetric Gratings}

In this experiment, the nontarget stimuli consisted of compound gratings with a $270^{\circ}$ phase angle; the targets had a $90^{\circ}$ phase angle. Unlike the gratings used in Experiment 1 , these gratings did not differ in magnitude of local contrast, but were mirror images of each other (see Figure 1).

Means and standard deviations for the total number and latencies of correct responses are given in Table 2, broken down by visual fields and the three exposure durations. The ANOVAs for both dependent variables revealed no significant VHF effects, exposure duration effects, or interactions. 
Table 2

Discrimination of Compound Gratings with $90^{\circ}$ and $270^{\circ}$ Phase Relationship

\begin{tabular}{lccc}
\hline \multicolumn{4}{c}{ Exposure Durations } \\
\cline { 2 - 4 } & $20 \mathrm{msec}$ & \multicolumn{3}{c}{$50 \mathrm{msec}$} & $150 \mathrm{msec}$ \\
\hline \multicolumn{4}{c}{ Correct Identifications } \\
LVF & $7.46-3.93$ & $7.96-3.32$ & $8.38-4.04$ \\
RVF & $7.79-4.31$ & $7.58-2.95$ & $8.08-3.41$ \\
\multicolumn{4}{c}{ Response Latencies } \\
LVF & $723-158$ & $722-155$ & $776-152$ \\
RVF & $732-193$ & $695-155$ & $811-183$ \\
\hline
\end{tabular}

Note-The procedure was as in Experiment 1 (see Table 1).

In conclusion, the discrimination of compound gratings with $90^{\circ}$ and $270^{\circ}$ phase angle was at chance level in all conditions. Therefore, a visual field effect could not be demonstrated.

\section{DISCUSSION}

In much the same way as Rentschler and Treutwein (1985) have compared the abilities of foveal and extrafoveal vision to distinguish grating pairs with identical power spectra (i.e., global image energy) and same separation in phase, we compared the abilities of the two hemispheres instantly to discriminate such stimulus pairs. Consistent with the observations of Fiorentini and Berardi (1984), a LVF-RH advantage was found for the non-mirror-image pair $\left(0^{\circ}\right.$ vs. $\left.180^{\circ}\right)$; the mirror-symmetrical pair $\left(90^{\circ}\right.$ vs. $270^{\circ}$ ) could not be discriminated at all.

Since the compound gratings used in our study had identical power spectra, their discrimination cannot be explained in terms of a bank of narrow-band spatial frequency channels (for a more thorough discussion, see Graham \& Nachmias, 1971). A model incorporating spatial frequency channels in conjunction with "phasesensitive devices" (Atkinson \& Campbell, 1974) is also inappropriate inasmuch as it would hardly account for the greatly different performance with the two stimulus pairs that had identical separation in phase (see also Rentschler \& Treutwein, 1985). Thus, we conclude that neither Fiorentini and Berardi's data nor ours can be explained in terms of global Fourier components of the stimulus patterns (i.e., their spatial frequencies, amplitudes or contrast values, and phase values). This implies that the discrimination of the grating pairs must depend on the neural processing of local image components or features of the luminance profiles.

Rentschler and Treutwein (1985) have proposed that two different processes underlie the rapid discrimination of compound gratings. One process captures contrast variability, but ignores positional relationships between image components. The registration of contrast variability is sufficient for explaining the discrimination of nonmirror-image gratings, that is, of grating pairs that differ in local contrast densities (in the present study $0^{\circ}$ vs. $\left.180^{\circ}\right)$. A possible model for this process would seem to be spatial correlation as used in image registration (Pratt, 1978). Furthermore, the registration of contrast variabilities occurs both in foveal and extrafoveal vision. Thus, the LVF advantage found by Fiorentini and Berardi (1984) and ourselves may be ascribed to a hemispheric asymmetry in the sensitivities of underlying mechanisms. The other process registers the positional relationships of image components and therefore mediates the quality of form. Rentschler and Treutwein (1985) found this process, which allows the discrimination of mirror-symmetrical grating pairs, ineffective at $2^{\circ}$ eccentricity in the RVF. The present results show that it is ineffective at $2^{\circ}$ both in the RVF and the LVF, thus supporting Rentschler and Treutwein's conclusion of a general loss of the relative position of local image components in extrafoveal vision.

A somewhat different interpretation of the previous results of Rentschler and Treutwein (1985) has been given by Watt (1985). It relates to the fact that in non-mirrorsymmetric pairs there is an immediate difference of organization of the stripes between the two patterns, whereas in mirror-symmetric pairs scrutiny is required to spot differences in relative position. Hence, Watt suggested that the elements of the perceptual representation of the patterns corresponded to their stripes. In the case of the stimuli used in the present study, immediate representation would be sufficient to allow for discrimination between patterns with single and double striation (e.g., between $180^{\circ}$ and $0^{\circ}$ compounds). These patterns may be considered different with respect to their "Gestalt" quality. Only a more refined representation would note differences in the relative positions of single dark lines, thus giving rise to discrimination of the $90^{\circ}$ and $270^{\circ} \mathrm{com}-$ pounds. As confirmed by the present data, the refinements of the latter representation are not available in extrafoveal vision. From this, we may conclude that hemispheric differences in early visual processing do exist only with respect to the coarser immediate representation.

\section{REFERENCES}

AtKinson, J., \& CAMPBELl, F.W. (1974). The effect of phase on the perception of compound gratings. Vision Research, 20, 391-396.

BADCOCK, D. R. (1984). Spatial phase or luminance profile discrimination? Vision Research, 24, 613-623.

BEAUMONT, J. G. (Ed.) (1982). Divided visual-field studies of cerebral organization. London: Academic Press.

Brettel, H., Caelli, T., Hizz, R., \& Rentschler, I. (1982). Modelling perceptual distortion: Amplitude and phase transmission in the human visual system. Human Neurobiology, 1, 61-67.

BurR, D. C. (1980). Sensitivity to spatial phase. Vision Research, 20, 159-162.

CaElli, T., Bevan, P. (1982). Visual sensitivity to two-dimensional spatial phase. Journal of the Optical Society of America, 72, 1375-1381.

Caelli, T. M., Hübner, M., Rentschler, I. (1985). The detection of phase shifts in two-dimensional images. Perception \& Psychophysics, 37, 536-542.

CaElli, T., Julesz, B., \& Gilbert, E. (1978). On perceptual analyzers 
underlying visual texture discrimination: Part II. Biological Cybernetics, 29, 201-214.

Fiorentini, A., \& Berardi, N. (1980). Perceptual learning specific for orientation and spatial frequency. Nature, 287, 43-44.

FIORENTINI, A., \& BERARDI, N. (1981). Learning in grating waveform discrimination: Specificity for orientation and spatial frequency. $V i-$ sion Research, 21, 1149-1158.

Fiorentini, A., \& Berardi, N. (1984). Right hemispheric superiority in the discrimination of spatial phase. Perception, 13, 695-708.

Gayl, I. E., Strasburger, H., Rentschler, I., \& Hilz, R. (1982). Evoked potentials and psychophysics of spatial phase sensitivity. Journal of the Optical Society of America, 72, 68.

Goodman, J. W., \& Silvestri, A. M. (1970). Some effects of Fourierdomain phase quantization. IBM Journal of Research and Development, 14, 478-484.

GraHaM, N., \& NaChmias, J. (1971). Detection of grating patterns containing two spatial frequencies: A comparison of single channel and multichannel models. Vision Research, 11, 251-259.

LAWDEN, M. C. (1982). The analysis of spatial phase in amblyopia. Human Neurobiology, 1, 55-60.

LAWDEN, M. C., (1983). An investigation of the ability of the human visual system to encode spatial phase relationships. Vision Research, 23, 1451-1463.

Lawden, M. C., Hess, R. F., \& Campbell, F. W. (1982). The discriminability of spatial phase relationships in amblyopia. Vision Research, 22, 1005-1016.

Nachmias, J., \& Weber, A. (1975). Discrimination of simple and complex gratings. Vision Research, 15, 217-223.

OPPENHEIM, A., \& LIM, P. (1981). The importance of phase in signals. Proceedings IEEE, 69, 529-541.

Pratt, W. (1978). Digital image processing. New York: Academic Press.

Rentschler, I., \& Treutwein, B. (1985). Loss of spatial phase relationships in extrafoveal vision. Nature, 313, 308-310.

WATT, R. J. (1985). Structured representation in low-level vision. $\mathrm{Na}$ ture, 313, 266-267.

Weiss, C., Rentschler, I., \& Caelli, T. (1985). Amblyopic processing of position information. Part II: Sensitivity to phase distortion. Experimental Brain Research, 60, 279-288.

(Manuscript received November 20, 1985; revision accepted for publication February 21, 1986.) 